\title{
Assessment of Resolution Parameters for CID-Based Shotgun Proteomic Experiments on the LTQ-Orbitrap Mass Spectrometer
}

\author{
Min-Sik Kim, ${ }^{\text {a,b }}$ Kumaran Kandasamy, ${ }^{\text {a,d }}$ Raghothama Chaerkady,, a,d and \\ Akhilesh Pandey ${ }^{\mathrm{a}, \mathrm{b}, \mathrm{c}}$ \\ a McKusick-Nathans Institute of Genetic Medicine, Baltimore, Maryland, USA \\ ${ }^{\mathrm{b}}$ Department of Biological Chemistry, Johns Hopkins University School of Medicine, Baltimore, Maryland, USA \\ ${ }^{c}$ Department of Pathology and Oncology, Johns Hopkins University School of Medicine, Baltimore, \\ Maryland, USA \\ ${ }^{\mathrm{d}}$ Institute of Bioinformatics, International Technology Park, Bangalore, India
}

Shotgun proteomics has been used extensively for characterization of a number of proteomes. High-resolution Fourier transform mass spectrometry (FTMS) has emerged as a powerful tool owing to its high mass accuracy and resolving power. One of its major limitations, however, is that the confidence level of peptide identification and sensitivity cannot be maximized simultaneously. Although it is generally assumed that higher resolution is better for peptide identifications, the precise effect of varying resolution as a parameter on peptide identification has not yet been systematically evaluated. We used the Escherichia coli proteome and a standard 48 protein mix to study the effect of different resolution parameters on peptide identifications in the setting of a shotgun proteomics experiment on an LTQ-Orbitrap mass spectrometer. We observed a higher number of peptide-spectrum matches (PSMs) whenever the MS scan was carried out by FT and the MS/MS in the ion-trap (IT) with the maximum PSMs obtained at an MS resolution of 30,000. In contrast, when samples were analyzed by FT for both MS and MS/MS, the number of PSMs was significantly lower $(\sim 40 \%$ compared with FT-IT experiments) with the maximum PSMs obtained when both the MS and MS/MS resolution were set to 15,000 . Thus, a $15 \mathrm{~K}-15 \mathrm{~K}$ resolution setting may provide the best compromise for studies where both speed and accuracy such as high-throughput posttranslational analysis and de novo sequencing are important. We hope that our study will allow researchers to choose between different resolution parameters to achieve their desired results from proteomic analyses. (J Am Soc Mass Spectrom 2010, 21, 1606-1611) (C) 2010 American Society for Mass Spectrometry

$\mathrm{O}$ ver the past decade, shotgun proteomics has become one of the most popular techniques in proteomics. Most recently, the LTQ-Orbitrap mass spectrometer [1], one of high-resolution Fourier transform mass spectrometers (FTMS), has been applied to a diverse set of experiments ranging from metabolomic analyses to clinical proteomics [2]. In an Orbitrap analyzer, the ions can be detected with a high mass accuracy of less than two parts per million (ppm) using internal standards and $5 \mathrm{ppm}$ with external calibration [3]. The maximum resolving power exceeds 100,000 at $400 \mathrm{~m} / \mathrm{z}$ [3], with additional features such as a wide dynamic range, fast duty cycle, and high sensitivity. These advantages allow complex peptide mixtures to be analyzed in greater depth with high confidence [4-6].

The various fragmentation methods available in LTQ-Orbitrap include collision induced dissociation

Address reprint requests to Dr. A. Pandey, McKusick-Nathans Institute of Genetic Medicine and Departments of Biological Chemistry, Pathology, and Oncology, Johns Hopkins University School of Medicine, Baltimore, MD 21205, USA. E-mail: pandey@jhmi.edu
(CID), electron-transfer dissociation (ETD) [7], and relatively newer methods such as pulsed-Q-dissociation (PQD) [8], high-energy C-trap dissociation, and higher energy collision dissociation (HCD) [9]. In most shotgun proteomic experiments, the important goal is to achieve a deeper proteome coverage, which can be accomplished by the above mentioned fragmentation methods. In recent years, there have also been several efforts to combine two fragmentation methods such as ECD-CID [10], CID-ETD [11], CID-PQD [12], and CIDHCD [13] to maximize the number of identified and quantitated peptides. The most popular method, however, for obtaining peptide sequence information remains CID based fragmentation. In MS-based proteomic analyses, tandem mass spectrometry is one of the conventional methods in which gaseous peptide ions are analyzed in data-dependent acquisition (DDA) [14] mode, where one MS scan for detecting precursors is followed by data dependent MS/MS scans for fragments generated by CID. The combined information from MS and MS/MS scans is used to search tandem 
spectra against protein sequence database using search algorithms such as Mascot [15], SEQUEST [16], OMSSA [17], X!Tandem [18], and Spectrum Mill [11].

There are a multitude of factors that directly affect the quality and quantity of the MS/MS spectra, which in turn influence the peptide identifications in proteomic analyses. Given the popularity of the LTQ mass spectrometer and the hybrid LTQ-FTMS, it is essential to systematically optimize the factors controlling the mass spectrometer. Although a number of parameters that could influence the peptide identification rate have been studied, including signal threshold of precursors for data dependent scans [19] and dynamic exclusion duration [20], the impact of varying resolution parameters has not been systematically.

To systematically evaluate the effect of resolution parameters in shotgun proteomic experiments, we used the Escherichia coli (E. coli) proteome as a model system with different MS and MS/MS resolution combinations. E. coli tryptic digests were fractionated by strong cation exchange chromatography and analyzed on an LTQOrbitrap mass spectrometer coupled to a nanoflow reversed-phase liquid chromatography system. The spectra were acquired in the Orbitrap mass analyzer at five different resolution settings for MS and in the LTQ or Orbitrap mass analyzer for MS/MS. We performed 100 LC-MS/MS runs using different combinations of resolution settings (each 'setting' being a specific resolution for MS in combination with a specific resolution for MS/MS) in the DDA mode. Using over 700,000 spectra generated in this study, false discovery rates (FDR) were calculated using target/decoy searches and 1\% FDR was used as a cutoff value for comparison. The criteria for evaluating resolution parameters included a comparison of the number of total spectra and the total number of identified unique peptides, peptide-spectrum matches (PSMs), and unique proteins. Finally, five different FT-IT resolution settings were explored in greater detail to evaluate the resolution settings for an optimal LC-MS/MS analysis using a 48 standard protein mixture.

\section{Materials and Methods}

\section{Sample Preparation and Processing}

Escherichia coli MG1655 (E. coli) cultures were grown overnight at $37^{\circ} \mathrm{C}$ in LB medium (MP Biomedicals, Solon, $\mathrm{OH}, \mathrm{USA})$. The cells were washed three times in sterile phosphate buffered saline (PBS) at room temperature and transferred to Dulbecco's modified Eagle medium (cat. no. 31,053-028; Invitrogen, Grand Island, NY, USA) containing $0.584 \mathrm{~g} / \mathrm{L}$ of glutamine and 0.11 $\mathrm{g} / \mathrm{L}$ of sodium pyruvate and further cultured for $4 \mathrm{~h}$. The cells were centrifuged, washed once with PBS, and the pellet was lysed by sonicating in the presence of $0.5 \%$ sodium dodecyl sulfate (SDS). Lysates were cleared by centrifugation at $20,000 \mathrm{~g}$ for $15 \mathrm{~min}$, and the supernatant was used for proteomic analysis. Protein concentration was determined using Lowry's method. Protein reduction was carried out using $5 \mathrm{mM}$ dithiothreitol at $60{ }^{\circ} \mathrm{C}$ for $20 \mathrm{~min}$ and subsequently alkylated with $10 \mathrm{mM}$ iodoacetamide in the dark at room temperature for $10 \mathrm{~min}$. In-solution digestion was carried out using trypsin protease (1:50 ratio in $0.01 \%$ SDS final; Promega, Madison, WI, USA) overnight at $37^{\circ} \mathrm{C}$ [11]. The tryptic digests were acidified by addition of formic acid $(0.1 \%$ final concentration). The peptides were fractionated on a strong cation exchange (SCX) column (2.1 $\mathrm{mm} \times 200 \mathrm{~mm}, 200 \AA$ pore, PolySULFOETHYL A; PolyLC Inc., Columbia, MD, USA) with a mobile phase of $25 \%$ acetonitrile, $10 \mathrm{mM} \mathrm{KH}_{2} \mathrm{PO}_{4} \mathrm{pH} 2.85$ (Buffer A), and five fractions were generated by increasing the salt gradient up to $350 \mathrm{mM} \mathrm{KCl}$ in Buffer A [21]. A 48 standard protein mixture (UPS1; Sigma-Aldrich, St. Louis, MO, USA) was digested using trypsin as described earlier [11].

\section{LC-MS/MS Experiments}

Each SCX fraction was analyzed on an LTQ-Orbitrap XL ETD mass spectrometer (Thermo, San Jose, CA, USA) at several resolution combinations. Approximately $1 \mu \mathrm{g}$ of peptides were trapped on a $2 \mathrm{~cm}$ long trap column packed with $\mathrm{C}_{18}$ material (5 $\mu \mathrm{m}$ and $300 \AA$ pore, Jupiter; Phenomenex, Torrance, CA, USA) with 5 $\mu \mathrm{L} / \mathrm{min}$ of flow rate at $99 \%$ Solvent $\mathrm{A}(0.1 \%$ formic acid in $\mathrm{H}_{2} \mathrm{O}$ ) and separated in $10 \mathrm{~cm}$ analytical column packed with $\mathrm{C}_{18}$ materials $(5 \mu \mathrm{m}$ and $300 \AA$ pore, Jupiter, Phenomenex) by gradient from $10 \%$ Solvent $\mathrm{B}$ (0.1\% formic acid in $90 \%$ acetonitrile) to $60 \%$ Solvent B for $65 \mathrm{~min}$, to $97 \%$ Solvent B for $9 \mathrm{~min}$, and to $90 \mathrm{~min}$ at $3 \%$ Solvent B. All the MS spectra were acquired on the Orbitrap while the data dependent MS/MS spectra were acquired on either the LTQ or the Orbitrap. Four most intense precursor ions from a survey scan within $\mathrm{m} / \mathrm{z}$ range from 400 to 1400 above 20,000 of intensity were isolated with a $4 \mathrm{Da}$ window and fragmented by CID with $35 \%$ normalized collision energy and the fragment ions were acquired at the profile mode with one microscan. The precursors were excluded, after fragmentation, for $90 \mathrm{~s}$ with a $0.02 \mathrm{Da}$ window. Maximum ion injection times were set to $10 \mathrm{~ms}$ for MS and $100 \mathrm{~ms}$ for MS/MS. The automatic gain control targets were set to $5 \times 10^{5}$ for MS in the Orbitrap, $1 \times 10^{4}$ for $\mathrm{MS}^{n}$ in the LTQ and $2 \times 10^{5}$ for $\mathrm{MS}^{n}$ in the Orbitrap. For further FT-IT comparison, peptides from a 48 protein mix digest were separated on a homemade Magic AQ $\mathrm{C}_{18}$ column $(10 \mathrm{~cm} \times 75 \mu \mathrm{m}, 5 \mu \mathrm{m}$ and $100 \AA$ pore; Michrom Bioresources, Inc., Auburn, CA, USA) including a $2 \mathrm{~cm}$ Magic AQ $C_{18}$ trap column. LC-MS/MS data were acquired in a data dependent mode in which 4 most intense precursor ions were isolated (width $\mathrm{m} / \mathrm{z}=4$ ) for CID with $35 \%$ normalized collision energy and detected in LTQ. The automatic gain control targets were set to $1 \times 10^{6}$ for MS in the Orbitrap and $1 \times 10^{4}$ for $\mathrm{MS}^{\mathrm{n}}$ in the LTQ. 


\section{Data Analysis}

Statistics of acquired spectra. The total number of MS and MS/MS scans was directly counted from the raw data by summing the number of scans from five SCX fractions. There were 208,863 and 240,387 spectra acquired in FT for MS and MS/MS, respectively, and 115,356 and 149,078 spectra acquired in FT for MS and in IT for MS/MS, respectively, resulting in a total of 713,684 spectra. In a separate FT-IT experiment, 15 LC-MS/MS analyses using standard proteins yielded a total of 168,873 spectra including 65,241 MS spectra in FT and 103,632 MS/MS spectra in IT.

Database search and processing. Tandem mass spectra were extracted from raw files using Mascot Daemon (ver. 2.2.2) with mass range from 600 to $5000 \mathrm{Da}$. The processed spectral data were then searched against $E$. coli protein database (4526 sequences and 1431,860 residues) using Mascot search algorithms (ver. 2.2.0). The data from 48 protein mix was searched against IPI human protein database $(148,380$ sequences and $62,526,836$ residues). In each case a reversed database was used to determine the false discovery rates (FDR). The search criteria included trypsin as protease with a maximum of two missed cleavages allowed. Carbamidomethylation at cysteine was set as a fixed modification, while deamidation at asparagine and glutamine, and oxidation at methionine, were set as variable modifications. Mass tolerance of $\pm 30 \mathrm{ppm}$ for precursor ions and 0.5 and $0.05 \mathrm{Da}$ for fragments detected using the LTQ and the Orbitrap respectively, were used in database search. The search results were downloaded from the Mascot server and processed using in-house python scripts for the estimation of FDR and the extracted data were stored in a MySQL database for subsequent analyses. As described previously, false discovery rates were calculated by dividing the number of PSMs from forward database search by the number of PSMs from reverse database search above a given score [22, 23].

Scan cycle times. For calculating the time acquired for one scan cycle (i.e., the time taken to acquire one MS scan and four subsequent MS/MS scans), we first counted the number of scans acquired in a $10 \mathrm{~min}$ window in the middle of the LC-MS/MS experiment, where resolution setting for both MS and MS/MS were same. Hence, average scan time is calculated by dividing $600 \mathrm{~s}$ by number of scans. Finally, the scan cycle time for each resolution combination was calculated based on the single scan times at the corresponding resolution.

\section{Data Availability}

These raw data associated with this manuscript have been submitted to Tranche and are downloadable from the ProteomeCommons.org website (https://proteomecommons. org/tranche/) using the following hash:
ROWCCrxC7ic21GeOrFnT/vKd/A/NwYLPjmfSu5iWI dIGMgciGNqEsHcGrU0XLTVjEvdPIWzOJlzq1CEwKOV wQbEgyMcAAAAAAABjHQ = =

The processed data, along with the search results, have also been submitted to the NCBI peptide data resource, Peptidome [24], and can be accessed using the URL http:// www.ncbi.nlm.nih.gov/peptidome/repository/PSE126/.

\section{Results and Discussion}

\section{Selection of Resolution Combinations for MS and $M S / M S$}

LTQ-Orbitrap mass spectrometer allows detection of gaseous ions and their fragments either in the linear ion trap (IT) or the Orbitrap (FT), where FT allows five different resolutions of 7500 to 100,000 in both MS and MS/MS. We decided to test 20 different combinations of resolution parameters during data dependent acquisition such that the MS/MS resolution does not exceed that of MS. Indeed, in the context of a shotgun proteomic experiment, high-resolution MS/MS spectra are of little use if the MS spectra themselves are acquired at low-resolution. As shown in Figure 1, 15 combinations from FT for MS and FT for MS/MS were chosen from the maximum of 25 theoretical combinations such that the resolution at the MS/MS level was always the same or lower than the setting for MS. In addition, five combinations of FT for MS and IT for MS/MS were also used to compare the influence of the resolution. For example, the resolution of 100,000 for MS can be combined with the resolutions of 100,000, 60,000, 30,000, 15,000, and 7500 for MS/MS, which will be referred to as 100-100, 100-60, 100-30, 100-15, and 100-7, respectively, in this article. Each combination of parameters was used to run five SCX fractions as a set, thereby

\section{Resolution settings}

\begin{tabular}{|c|c|c|c|c|c|c|}
\hline \multirow{5}{*}{$\begin{array}{c}\text { Orbitrap } \\
\text { (FT) }\end{array}$} & 100,000 & & & & & $100-100$ \\
\hline & 60,000 & & & & $60-60$ & $100-60$ \\
\hline & 30,000 & & & $30-30$ & $60-30$ & $100-30$ \\
\hline & 15,000 & & $15-15$ & $30-15$ & $60-15$ & $100-15$ \\
\hline & 7,500 & 7-7 & $15-7$ & $30-7$ & $60-7$ & $100-7$ \\
\hline \multicolumn{2}{|c|}{ Ion Trap (IT) } & 7-IT & 15-IT & 30-IT & 60-IT & 100-IT \\
\hline \multirow[t]{2}{*}{ MS/MS } & & 7,500 & 15,000 & 30,000 & 60,000 & 100,000 \\
\hline & & \multicolumn{5}{|c|}{ Orbitrap (FT) } \\
\hline
\end{tabular}

Figure 1. Resolution combinations chosen for this study. There are 36 possible resolution combinations in MS and MS/MS in an LTQ-Orbitrap mass spectrometer. Of these 36 possible combinations, 20 were selected for comparison purposes in this study. For example, 60-15 indicates that the resolution of 60,000 for MS and 15,000 for MS/MS was employed to acquire data-dependent MS/MS spectra. 
resulting in a total of 100 LC-MS/MS runs for comparison purposes. The data were searched against an E. coli database using the Mascot search algorithm.

\section{The Number of MS/MS Scans is Inversely Related to the Scan Cycle Time}

The number of scans differed considerably at various MS resolution parameters and with respect to detection methods (IT versus FT) as shown in Figure 2. For example, the difference in the number of MS/MS spectra ranges from 1.2-fold between 100-IT (lowest in FT-IT experiments) and 15-7 (highest in FT-FT experiment) to 5-fold between 100-100 (lowest in FT-FT experiments) and 15-IT (highest in FT-IT experiments). The number of MS/MS spectra was similar in FT-IT experiments $[\sim 8.4 \%$ relative standard deviation (RSD)], while they varied greatly in FT-FT experiments ( $\sim 30 \%$ RSD). As expected, the number of MS/MS scans acquired at lower MS/MS resolution either by FT or IT was greater than those acquired at higher resolution. This is because of the fact that increasing resolution increases scan duration. As described in data analysis section, acquiring scans at resolution of 7500, 15,000,30,000,60,000, or 100,000 took, on average, 0.44, 0.50, 0.62, 1.00, and $1.78 \mathrm{~s}$, respectively. Based on these estimates, scan cycle times at different resolution combinations (i.e., one scan cycle consisting of one MS scan followed by four MS/MS scans) was calculated. For example, one scan cycle took $8.9 \mathrm{~s}$ at $100-100$, while only $1.2 \mathrm{~s}$ were used in one scan cycle at 7-IT. Thus, the scan cycle times at 100-100 are $\sim 7$-fold, $\sim 4$-fold, $\sim 3$-fold, and $\sim 2$-fold longer than those at 7-IT, 7-7, 60-15, and 100-30, respectively. The scan cycle times were similar at 7-7, 15-7, 15-15, and $30-7$ and quite short (1.2 1.8 s) for 7-IT,

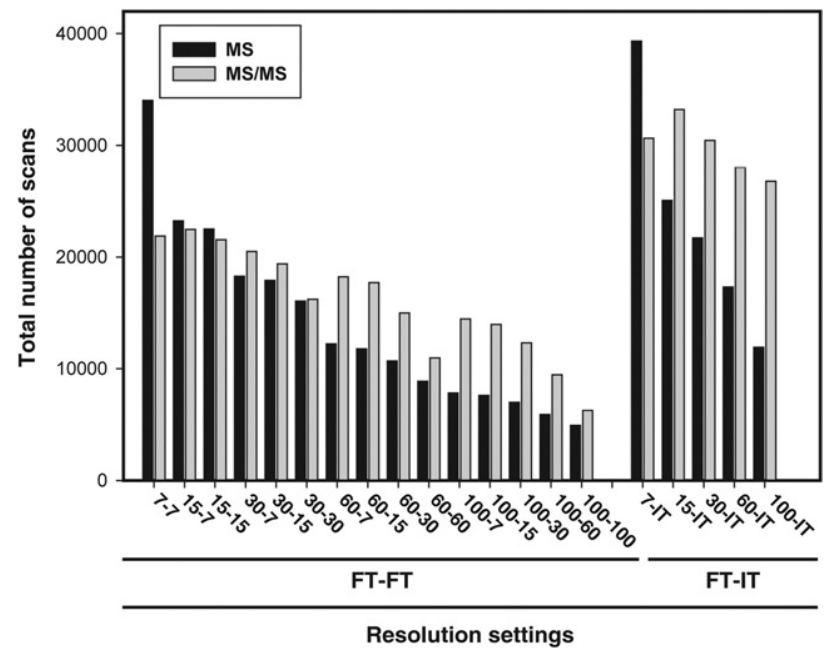

Figure 2. Total number of MS and MS/MS scans. Total number of MS and MS/MS scans was directly counted from each raw file. The number of MS scans varied greatly at different resolution combinations ranging from $\sim 5000(100-100)$ to $\sim 40,000$ (7-IT) while those of MS/MS scans ranged from $\sim 6000$ (100-100) to $\sim 33,000$ (15-IT)

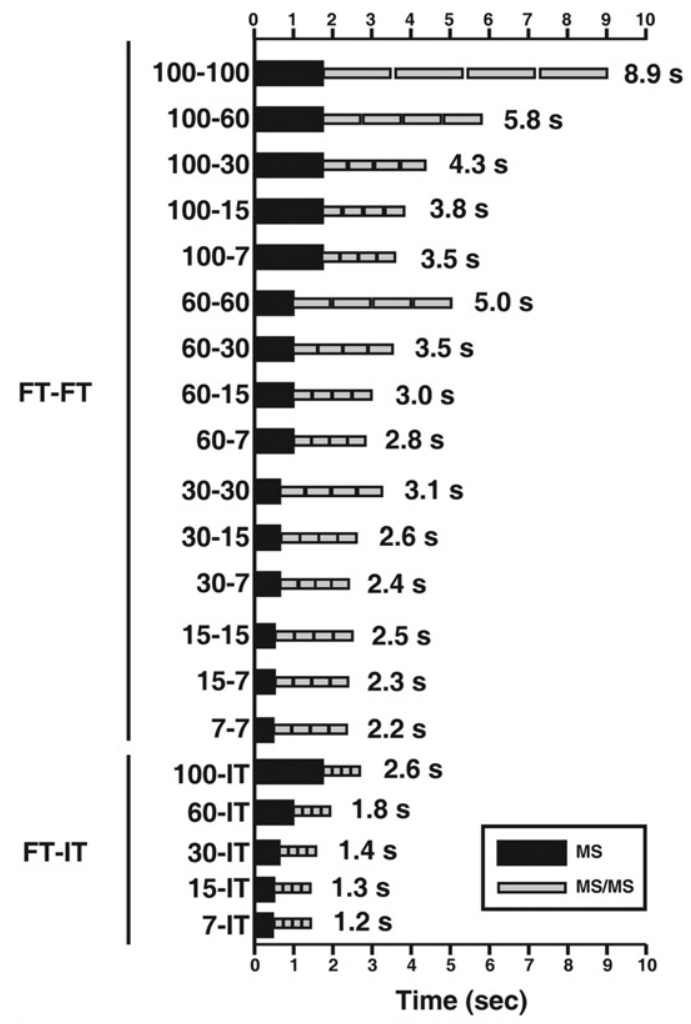

Figure 3. Scan cycle times for FT-IT and FT-FT experiments. The scan cycle times (i.e., one MS scan and the subsequent four MS/MS scans) were calculated based on actual scan times at resolutions of 7-7, 15-15, 30-30, 60-60, and 100-100 resulting in total scan cycle times of $0.44,0.50,0.62,1.00$, and $1.78 \mathrm{~s}$, respectively. The slowest scan cycle time was observed at 100-100, while the fastest scan cycle times were observed at 7-IT, 15-IT, 30-IT, and 60-IT.

15-IT, 30-IT, and 60-IT as shown in Figure 3. As expected, it was observed that the scan cycle time was inversely proportional to the number of MS/MS scans.

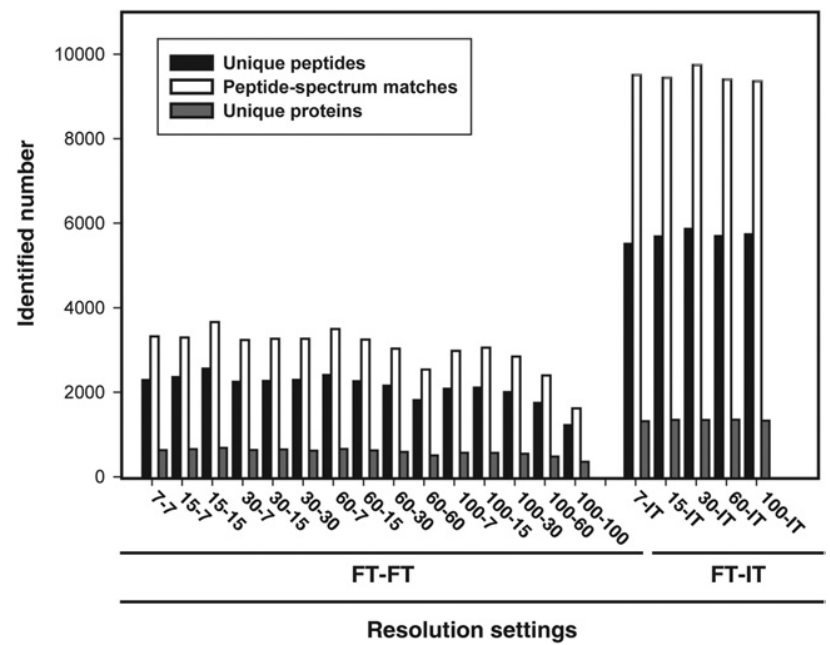

Figure 4. Distribution of the number of unique peptides, peptidespectrum matches, and unique proteins. The number of unique peptides, peptide-spectrum-matches (PSMs), and unique proteins is shown at the indicated resolution combinations at $1 \%$ FDR. 
Resolution Settings Yielding the Highest Number of Peptide-Spectrum Matches, Unique Peptides, and Proteins

The total number of unique peptides, peptide-spectrummatches (PSMs), or unique proteins identified at $1 \%$ FDR was examined at all resolution combinations (Figure 4). In FT-FT experiments, the number of unique peptides ranged from 1264 at $100-100$ to 2603 at $15-15$ and PSMs from 1663 at $100-100$ to 3705 at $15-15$. The number of unique proteins identified was 398 at 100-100 and 725 at 15-15. In FT-IT experiments, the number of unique peptides was 5554 at 7-IT and 5909 at 30-IT, while the number of PSMs was 9401 at 100-IT and 9789 at 30 -IT. The number of unique proteins ranged from 1356 at 7 -IT to 1393 at 60 -IT. The number of unique peptides, PSMs, and unique proteins exhibited a greater variation in the FT-FT experiments, while a similar number of unique peptides, PSMs, and unique proteins were identified across all the FT-IT experiments. To better assess whether the differences in the identification between FT-FT group and FT-IT group were sta-

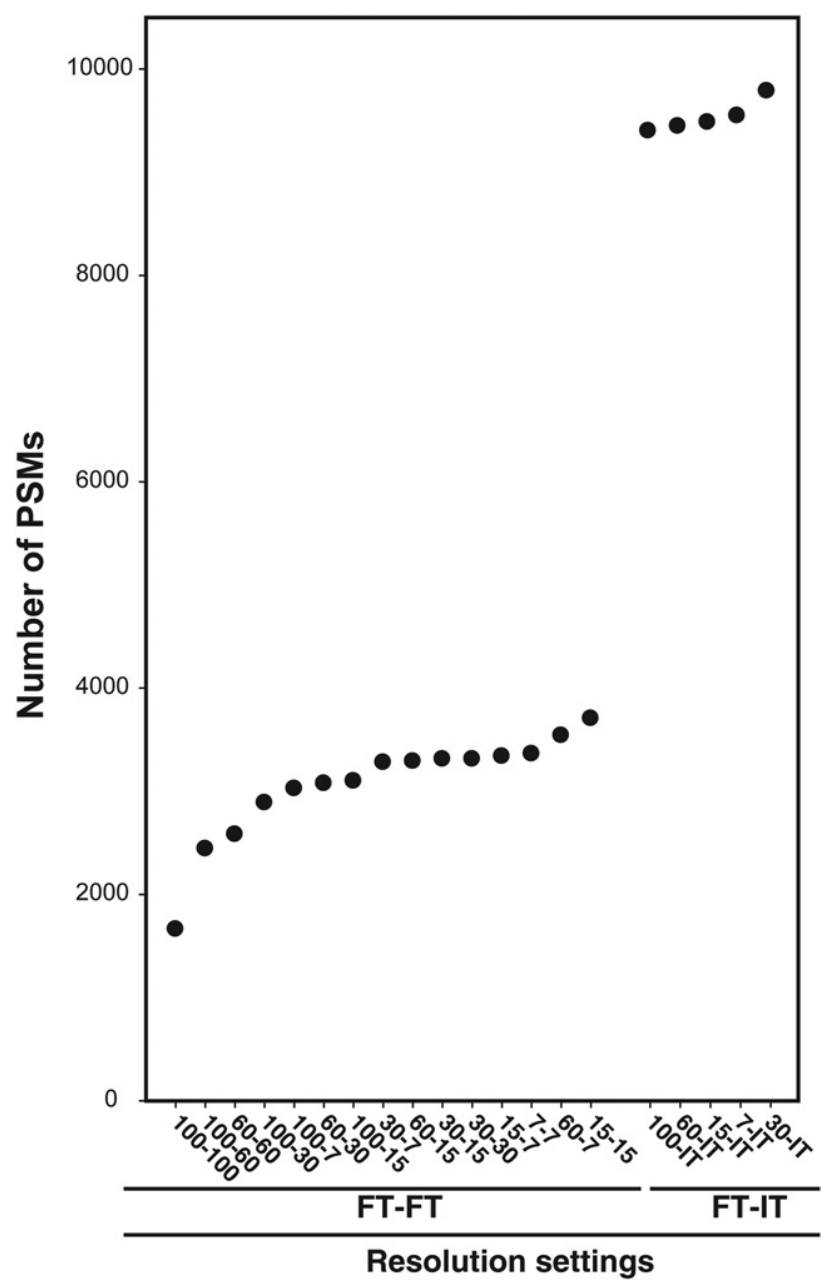

Figure 5. Ordered resolution combinations by the number of peptide-spectrum matches. The number of PSMs identified at 1\% FDR was used to order resolution combinations.

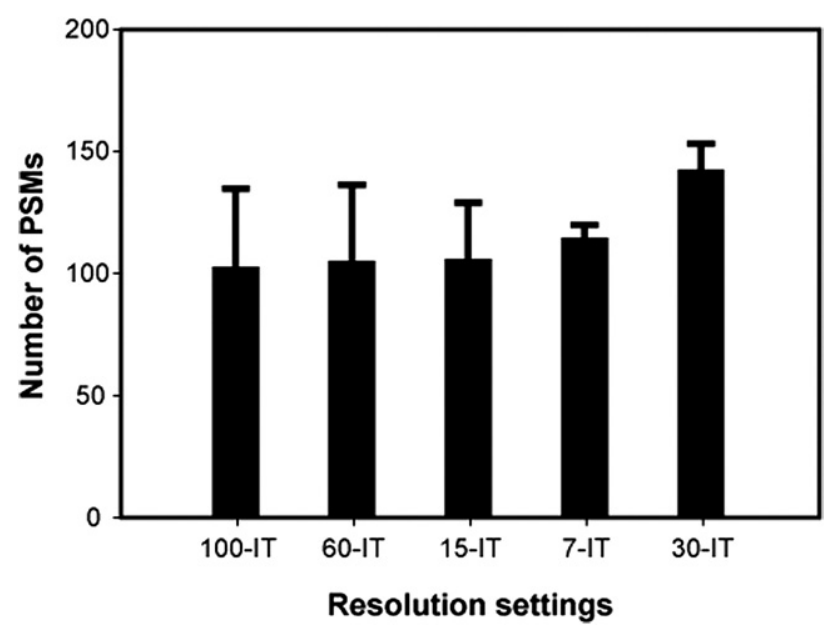

Figure 6. Comparison of different resolution settings in FT-IT experiments. The number of PSMs at different resolution settings at $1 \%$ FDR using the 48 protein standard mixture is shown. Error bars denote standard deviations of the number of PSMs obtained in each case.

tistically significant, we performed a two-tailed unpaired $t$-test. We observed that the number of PSMs in FT-FT group was significantly lower than that observed in FT-IT group $\left(P\right.$ value $\left.=1.8 \times 10^{-16}\right)$. This can be explained by the fact that IT detection is more sensitive than FT detection because of faster scan speed and lower detection threshold in the ion-trap [25]. Although the difference in the number of PSMs obtained at different FT-IT settings was not dramatic (9401-9789 PSMs), there was a large variation in the number of PSMs at different FT-FT settings (1663-3705 PSMs) with the maximum number of PSMs observed at the 15-15 setting (Figure 5). Although, additional experiments will be required to evaluate this in greater detail, it seems that longer scan cycle times (e.g., 100-100, 100-60, and 60-60) result in a corresponding decrease in PSMs. Within the FT-IT group, we wished to determine if any resolution combination consistently led to a higher number of PSMs. Thus, we analyzed a 48 protein mix in triplicate at the five different FT-IT settings. We again observed that the maximum number of PSMs was obtained at an MS resolution of 30,000. This number was significantly higher $(P$ value $<0.05)$ than all other resolution settings except for 60-IT (Figure 6).

\section{Conclusions}

The impact of resolution parameters on the throughput of tandem mass spectrometry experiments was systematically assessed in this study. In our analysis, based on the number of unique peptides, PSMs, and unique proteins identified at $1 \%$ FDR from E. coli proteome, a higher number of identifications were obtained in the FT-IT experiments compared with FT-FT experiments. For maximizing the number of identifications, we found that 30-IT settings (closely followed by 60-IT setting) performed significantly better than other set- 
tings from a 48 protein mixture. Thus, we conclude that the rate of identification could be maximized at 30-IT setting for proteomic profiling studies. However, 15-15 setting may be preferable for applications such as high throughput post-translational modification analysis, proteogenomic studies, and de novo sequencing; where one seeks a compromise between the number of identifications and a high accuracy of identification.

\section{Acknowledgments}

The authors acknowledge support in part for this study by a grant S10RR023025 from the High End Instrumentation Program of the National Institutes of Health, a Department of Defense Era of Hope Scholar award (W81XWH-06-1-0428), an NIH roadmap grant for Technology Centers of Networks and Pathways (U54RR020839), and a contract N01-HV-28,180 from the National Heart Lung and Blood Institute.

\section{References}

1. Makarov, A. Electrostatic Axially Harmonic Orbital Trapping: A HighPerformance Technique of Mass Analysis. Anal. Chem. 2000, 72(6), 1156-1162.

2. Yates, J. R.; Ruse, C. I.; Nakorchevsky, A. Proteomics by Mass Spectrometry: Approaches, Advances, and Applications. Annu. Rev. Biomed. Eng. 2009, 11, 49-79.

3. Makarov, A.; Denisov, E.; Kholomeev, A.; Balschun, W.; Lange, O.; Strupat, K.; Horning, S. Performance Evaluation of a Hybrid Linear Ion Trap/Orbitrap Mass Spectrometer. Anal. Chem. 2006, 78(7), 2113-2120.

4. Clauser, K.; Baker, P.; Burlingame, A. Role of Accurate Mass Measurement $( \pm 10 \mathrm{ppm})$ in protein identification strategies employing MS or MS MS and Database Searching. Anal. Chem. 1999, 71(14), 2871-2882.

5. Zubarev, R.; Hakansson, P.; Sundqvist, B. Accuracy Requirements for Peptide Characterization by Monoisotopic Molecular Mass Measurements. Anal. Chem. 1996, 68(22), 4060-4063.

6. Conrads, T. P.; Anderson, G. A.; Veenstra, T. D.; Pasa-Tolic, L.; Smith, R. D. Utility of Accurate Mass Tags for Proteome-Wide Protein Identification. Anal. Chem. 2000, 72(14), 3349-3354.

7. Syka, J. E.; Coon, J. J.; Schroeder, M. J.; Shabanowitz, J.; Hunt, D. F. Peptide and Protein Sequence Analysis by Electron Transfer Dissociation Mass Spectrometry. Proc. Natl. Acad. Sci. U.S.A. 2004, 101(26), 9528-9533.

8. Griffin, T. J.; Xie, H.; Bandhakavi, S.; Popko, J.; Mohan, A.; Carlis, J. V.; Higgins, L. iTRAQ Reagent-Based Quantitative Proteomic Analysis on a Linear Ion Trap Mass Spectrometer. J. Proteome Res. 2007, 6(11), 4200-4209.
9. Olsen, J. V.; Macek, B.; Lange, O.; Makarov, A.; Horning, S.; Mann, M. Higher-Energy C-trap Dissociation for Peptide Modification Analysis. Nat. Methods 2007, 4(9), 709-712.

10. Bushey, J. M.; Baba, T.; Glish, G. L. Simultaneous Collision Induced Dissociation of the Charge Reduced Parent Ion during Electron Capture Dissociation. Anal. Chem. 2009, 81(15), 6156-6164.

11. Molina, H.; Matthiesen, R.; Kandasamy, K.; Pandey, A. Comprehensive Comparison of Collision Induced Dissociation and Electron Transfer Dissociation. Anal. Chem. 2008, 80 (13), 4825-4835.

12. Guo, T.; Gan, C. S.; Zhang, H.; Zhu, Y.; Kon, O. L.; Sze, S. K Hybridization of Pulsed-Q Dissociation and Collision-Activated Dissociation in Linear Ion Trap Mass Spectrometer for iTRAQ Quantitation. J. Proteome Res. 2008, 7(11), 4831-4840.

13. Kocher, T.; Pichler, P.; Schutzbier, M.; Stingl, C.; Kaul, A.; Hasenfuss, G.; Teucher, N.; Penninger, J.; Mechtler, K. High Precision Quantitative Proteomics Using iTRAQ on an LTQ Orbitrap: A New Mass Spectrometric Method Combining the Benefits of All. J. Proteome Res. 2009, 8(10), 4743-4752.

14. Wenner, B. R.; Lynn, B. C. Factors that Affect Ion Trap Data-Dependent MS/MS in Proteomics. J. Am. Soc. Mass Spectrom. 2004, 15(2), 150-157.

15. Perkins, D. N.; Pappin, D. J.; Creasy, D. M.; Cottrell, J. S. ProbabilityBased Protein Identification by Searching Sequence Databases Using Mass Spectrometry data. Electrophoresis 1999, 20(18), 3551-3567.

16. Eng, J.; McCormack, A.; Yates, J. An Approach to Correlate Tandem Mass-Spectral Data of Peptides with Amino-Acid Sequences in a Protein Database. J. Am. Soc. Mass Spectrom. 1994, 5(11), 976-989.

17. Geer, L. Y.; Markey, S. P.; Kowalak, J. A.; Wagner, L.; Xu, M.; Maynard D. M.; Yang, X.; Shi, W.; Bryant, S. H. Open Mass Spectrometry Search Algorithm. J. Proteome Res. 2004, 3(5), 958-964.

18. Craig, R.; Beavis, R. C. TANDEM: Matching Proteins with Tandem Mass Spectra. Bioinformatics 2004, 20(9), 1466-1467.

19. Wong, C. C. Cociorva, D.; Venable, J. D; Xu, T. Yates, J. R. III Comparison of Different Signal Thresholds on Data-Dependent Sampling in Orbitrap and LTQ Mass Spectrometry for the Identification of Peptides and Proteins in Complex Mixtures. J. Am. Soc. Mass Spectrom. 2009, 20(8), 1405-1414.

20. Zhang Y: Wen, Z; Washburn, M. P.; Florens, L. Effect of Dynamic Exclusion Duration on Spectral Count Based Quantitative Proteomics. Anal. Chem. 2009, 81(15), 6317-6326.

21. Chaerkady, R.; Harsha, H. C.; Nalli, A.; Gucek, M.; Vivekanandan, P.; Akhtar, J.; Cole, R. N.; Simmers, J.; Schulick, R. D.; Singh, S.; Torbenson, M.; Pandey, A.; Thuluvath, P. J. A Quantitative Proteomic Approach for Identification of Potential Biomarkers in Hepatocellular Carcinoma. J. Proteome Res. 2008, 7(10), 4289-4298

22. Elias, J. E.; Gygi, S. P. Target-Decoy Search Strategy for Increased Confidence in Large-Scale Protein Identifications by Mass Spectrometry. Nat. Methods 2007, 4(3), 207-214.

23. Kandasamy, K.; Pandey, A.; Molina, H. Evaluation of Several MS/MS Search Algorithms for Analysis of Spectra Derived from Electron Transfer Dissociation Experiments. Anal. Chem. 2009, 81(17), 7170-7180.

24. Slotta, D. J.; Barrett, T.; Edgar, R. NCBI Peptidome: A New Public Repository for Mass Spectrometry Peptide Identifications. Nat. Biotechnol. 2009, 27(7), 600-601.

25. Olsen, J. V.; Schwartz, J. C.; Griep-Raming, J.; Nielsen, M. L.; Damoc, E.; Denisov, E.; Lange, O.; Remes, P.; Taylor, D.; Splendore, M.; Wouters, E. R.; Senko, M.; Makarov, A.; Mann, M.; Horning, S. A Dual Pressure Linear Ion Trap Orbitrap Instrument with Very High Sequencing Speed. Mol. Cell. Proteom. 2009, 8, 2759-2769. 